\title{
Pratiques
}

Linguistique, littérature, didactique

\section{«Lire » et « étudier » avec l'écrit : des activités différentes pour les enfants dans le temps et dans l'espace social ; des inégalités renouvelées}

"Reading" and "studying" in writing: different activities for children in time and in social space; renewed inequalities

\section{Stéphane Bonnéry}

\section{OpenEdition Journals}

Édition électronique

URL : http://journals.openedition.org/pratiques/8426

DOI : $10.4000 /$ pratiques. 8426

ISSN : 2425-2042

Éditeur

Centre de recherche sur les médiations (CREM)

\section{Référence électronique}

Stéphane Bonnéry, « «Lire » et « étudier » avec l'écrit : des activités différentes pour les enfants dans le temps et dans l'espace social; des inégalités renouvelées », Pratiques [En ligne], 185-186 | 2020, mis en ligne le 30 juin 2020, consulté le 15 octobre 2020. URL : http://journals.openedition.org/pratiques/ 8426 ; DOI : https://doi.org/10.4000/pratiques.8426

Ce document a été généré automatiquement le 15 octobre 2020

(c) Tous droits réservés 


\title{
« Lire » et « étudier » avec l'écrit : des activités différentes pour les enfants dans le temps et dans l'espace social ; des inégalités renouvelées
}

\author{
"Reading" and "studying" in writing: different activities for children in time and \\ in social space; renewed inequalities
}

\section{Stéphane Bonnéry}

1 Une diversité de recherches sociologiques montrent que selon les classes sociales, s'il y a toujours des exceptions, la manière de lire est assez différente: focalisée sur la "participation " (adhésion au suspens, identification aux personnages, etc.) dans les classes populaires, plus réflexive en recourant à des outils « analytiques » pour irriguer la participation en retour dans les catégories plus cultivées (Lahire, 1993; Mauger, 1999 ; Renard 2002 ; Collovald \& Neveu, 2004). Les modalités plus populaires du lire ne sont pas moins dignes, mais elles sont en contradiction avec celles que l'école requiert pour accéder aux études longues. Quel est ce lire attendu pour l'étude ? Comment s'estil développé, et de quelles inégalités s'est-il accompagné?

2 Pour répondre à ces questions, cette contribution constitue la synthèse d'un programme de recherche, en donnant à voir la complémentarité de plusieurs enquêtes complémentaires dont les résultats n'ont été jusqu'ici publiés que partiellement et séparément. $\mathrm{Au}$ commencement, une recherche sur la construction des inégalités sociales d'apprentissage avait conduit à faire le constat que, chez des élèves du CM2 à la $5^{\mathrm{e}}$, l'activité que recouvre le terme «étudier » revêtait des sens différents, ce qui a des effets très différenciateurs sur le plan des apprentissages (Bonnéry, 2007). De plus, cette recherche nous avait également conduit à nous interroger sur un certain nombre de documents écrits (tableaux de synthèse, fiches d'activités, etc.) dont la lecture ne relevait pas de l'évidence et dont l'utilisation était très différenciatrice. Nous avions 
axé l'analyse sur l'écart entre les usages effectifs des différents types d'élèves et ceux attendus par les enseignants et l'institution scolaire. Cette approche avait l'avantage de pointer les enjeux sociaux des situations d'enseignement-apprentissage, notamment dans la confrontation des évidences scolaires que relaient les enseignants et celles des élèves des classes populaires. Mais elle laissait de côté ce qui, dans le contexte de la classe, guide ou contraint aussi bien l'action des enseignants que celle des élèves. Sur l'usage des documents écrits plus spécifiquement, une question émergeait : sachant que l'utilisation scolairement attendue était peu ou pas enseignée, ou bien de façon inopérante car disjointe des contenus de savoirs (Bautier, Bonnéry, \& Clément, 2017), comment les élèves qui utilisaient pertinemment ces documents y parvenaient-ils, tandis que d'autre non, alors qu'en principe ils étaient exposés aux mêmes programmes? L'explication se situait-elle dans la socialisation familiale et/ou dans l'expérience scolaire antérieure en maternelle et début d'élémentaire?

Un programme a été conduit en plusieurs volets complémentaires. Ces différentes enquêtes et corpus seront présentées successivement dans les parties correspondantes de notre propos. Après avoir présenté les principaux éléments du cadre théorique qui ont structuré l'approche commune à ces différents volets, nous montrerons d'abord que « lire » aujourd'hui, pour s'approprier l'histoire d'un album de jeunesse destiné aux 4-7 ans comme pour étudier des savoirs entre le CE2 et la $5^{\mathrm{e}}$ à l'aide d'un manuel, ne signifie plus la même chose qu'il y a 70 ans, et que ces ouvrages actuels sont utilisés de façon très inégale dans les classes selon les contextes sociaux. Nous verrons ensuite que quand les dispositifs d'enseignement ne créent pas toutes les conditions de l'apprentissage de ce lire bien spécifique attendu à l'école, alors les lectures d'albums dans les familles sont susceptibles d'expliquer les manières de lire quand les enfants grandissent, très inégales face aux manuels.

\section{Le cadrage codé des supports}

Le cadre théorique mobilisé s'inscrit principalement dans la sociologie de l'éducation, en articulant problématique des inégalités et problématique de la transmissionappropriation puisque l'objet de la recherche porte avant tout sur les manières de raisonner. C'est pourquoi nous avons choisi de désigner les manuels et albums utilisés par les enseignants et les élèves en tant que "supports ». Ce n'est pas contradictoire avec la conceptualisation en termes de «textes composites " (Bautier, Crinon, DelarueBreton \& Marin, 2012) ou "documents» (Tricot, Sahut \& Lemarié, 2016; Halté \& Maisonneuve dans l'introduction du présent dossier), ces dernières formulations mettant l'accent sur les logiques internes aux objets, même si leurs auteurs insistent sur la mise en correspondance des manières de lire que la forme de l'écrit autorise et de celles effectivement conduites. Ce dernier aspect nous semble essentiel, parce que "une même oeuvre est comprise différemment par des lecteurs distincts ", et parce que « deux oeuvres différentes ne mettent pas en jeu les mêmes processus de lecture [...] pour une même population ", ce qui conduit à "prendre en compte une oeuvre précise dans sa relation avec les lecteurs aux caractéristiques identifiées " (Segré, 2001), les études littéraires et sociologiques ayant le plus souvent tendance à privilégier un seul des deux aspects. Ainsi, à la différence des « théories de la réception » qui n'ont pas étudié les réceptions effectives (Mauger 1999), le «lecteur supposé », ou plutôt la 
«définition sociale de l'enfant» lecteur (Chamboredon \& Fabiani, 1977a et 1977b) objectivable dans le livre, est ici confrontée aux pratiques des lecteurs effectifs.

5 Les supports matériels écrits (manuels, fiches...) influencent en partie l'usage qu'en font les enseignants ou parents ainsi que les élèves. En effet, en jouant sur les mots, le manuel ou l'album sont tout d'abord le "support", au sens du réceptacle, de significations qui sont déposées, matérialisées dans l'écrit (Bonnéry, 2015a). Celles-ci peuvent être idéelles et conscientes chez leur auteur (une morale, une notion), mais elles peuvent aussi relever d'habitudes de pensée, par exemple dans un manuel d'histoire la mise à distance des discours des témoins historiques, dont les propos sont à confronter avec d'autres éléments qui corroborent, nuancent, ou infirment la version donnée.

6 Ainsi, simultanément, et en un second sens (plus sportif), le manuel ou l'album "supporte», encourage un usage attendu. Ces formules imagées d'activités " attendues", "supposées", "requises", si elles évoquent des demandes faites au lecteur, n'impliquent pas forcément un sujet pensant qui aurait formulé ces demandes. On désigne là ce qui est nécessaire pour accéder aux significations et aux activités de raisonnement les plus élaborées inscrites dans la matérialité du livre (l'organisation des éléments dans la page par exemple) et qui, justement parce que ce sont des définitions sociales latentes, s'imposent aux usagers du livre. Il s'agit d'une conception matérialiste du cadrage de l'activité de lecture, au sens où cette dernière est guidée par les « constituants matériels» de ces activités cognitives (Grossmann, 2006, p. 27), par la forme concrète de l'écrit socio-historiquement élaborée, et donc par les "pouvoirs cognitifs » et les «technologies intellectuelles » (Goody, 1978 [1977], 1986, 2006 [2000], 2007 ; Privat \& Kara, 2006 ; Bautier, Crinon, Delarue-breton \& Marin 2012 ; Joigneaux, 2009) que peut procurer le décodage des modes de raisonnements inscrits dans l'espace graphique et dans son organisation. C'est ce «cheminement intellectuel » que nous avons cherché à mettre au jour dans chacun des corpus qui sont étudiés. Mais ce cadrage, s'il contraint le périmètre des usages possibles, ne constitue pas un conditionnement mécanique : le support autorise plusieurs usages, par les adultes et les enfants, aux effets différents.

7 Dans un troisième et dernier sens, le livre tolère ou "supporte » donc plusieurs usages puisqu'on peut se saisir différemment du même album ou du même manuel. Les significations sont inscrites dans l'écrit selon un "code » qui implique un décodage pour accéder à celles-ci, et ce code est inégalement partagé, et accessible, selon le degré d'implicite dont il est porteur (Bourdieu, Passeron \& Saint-Martin, 1968). Ainsi lorsque le cadrage du support est faible, c'est-à-dire que ce dernier guide le lecteur par des signes assez discrets et implicites, il laisserait agir prioritairement les dispositions construites dans la socialisation antérieure : s'exerceraient donc des inégalités entre les lectures accédant aux significations les plus élaborées permise par l'ouvrage d'une part et les lectures hétérodoxes d'autre part, au sens où ces dernières sont éloignées du raisonnement lettré.

\section{Étudier ce que « lire » veut dire aujourd'hui}

8 É. Durkheim encourage la méthode comparative, pour que le chercheur puisse se décentrer des évidences de sa culture et de son époque, cette comparaison pouvant être 
synchronique, entre sociétés différentes, ou diachronique au sein d'une même société (Durkheim, 2007 [1893], 1999 [1938]).

9 Pour saisir ce que sont les exigences actuelles en matière de lecture attendue à l'école et dans les familles, nous les avons donc comparées avec celles en vigueur antérieurement. Nous avons constitué des corpus de manuels et d'albums de jeunesse parus en France depuis 1945, analysés selon les mêmes critères. Cette date de début permet à la fois d'éviter la période très spécifique de l'occupation et du régime de Vichy pour se concentrer sur les grandes évolutions de fond, et de commencer l'étude au moment où s'implantent les techniques importées des États-Unis qui rentabilisent les publications traduites et changent la fabrication d'ouvrage, conduisant à l'abaissement significatif du prix des livres, progressivement accessibles financièrement à une part croissante de familles.

10 Un premier corpus permet d'étudier l'évolution de 52 manuels scolaires, dans des périodes définies par les réformes de démocratisation progressive de l'accès aux études longues. Il s'agit de 10 manuels publiés de 1945 à 1958 (la réforme Berthoin datant de 1959), 12 édités entre 1960 et 1974 (réforme Haby en 1975), 12 parus entre 1976 et 1994 (avant les programmes de 1995, durant la massification du lycée et la création du lycée professionnel ${ }^{1}$ ), et 28 entre 1998 et 2013. Ils dont destinés aux classes du CE2 à la $5^{\mathrm{e}}$, pour que l'on puisse considérer qu'il s'adresse à un enfant relativement autonome dans le déchiffrage. Ce corpus est complété de 30 heures d'observation de l'utilisation des manuels de la dernière période dans des classes, pour moitié dans des établissements au recrutement socialement hétérogène et pour l'autre au recrutement essentiellement populaire. La comparaison synchronique vient donc compléter la première, diachronique pour saisir le « lire » attendu et encouragé aujourd'hui à l'école, selon à qui l'on enseigne.

11 Un deuxième corpus porte sur l'évolution des albums de littérature de jeunesse qui ont été publiés durant les mêmes périodes que les manuels (1945-1974; 1975-1994; 1995-2014) afin de voir si des parallèles existent entre l'évolution de l'écrit scolaire au regard des réformes de démocratisation, et l'évolution de l'écrit de fiction pour l'enfance. Les albums du corpus s'adressent de façon privilégiée aux enfants scolarisés en Grande section de maternelle (GSM) et CP (voire à la moyenne section de maternelle et au CE1), lorsque la lecture se fait de façon conjointe avec un adulte. Ce sont au total 450 albums narratifs (les abécédaires, documentaires, etc. n'ont pas été retenus) qui ont été étudiés avec différents sous-corpus répartis en quantité homogènes dans les différentes périodes: 205 albums avec un loup, et 58 avec un autre personnage traditionnellement considéré comme "méchant», 25 albums de la collection Babar Hachette grand format (la série francophone de la plus longue durée), 20 de la collection " premières histoires » du Père Castor (parus jusqu'en 1966), 52 albums de la collection «Un petit livre d'or » ou «Un petit livre d'argent " (parus jusque dans les années 1980, Boulaire, 2016), 270 albums divers représentant la pluralité (esthétique, politique, confessionnelle $v s$ laïque, artistique $v s$ éducative $v s$ marchande) d'éditeurs et d'auteurs durant les périodes. À cette comparaison diachronique, s'ajoute une autre, synchronique avec 123 familles (ayant un enfant de la tranche d'âge concernée), aux caractéristiques sociales variées, qui ont été observées sur des «lectures partagées » d'albums (Grossmann, 1996; Frier, 2006), pour saisir ce que «lire» signifie en acte, lecture après laquelle les parents ont été interviewés. 

grands éditeurs scolaires produisent à destination des familles dans leur tâche d'accompagnement scolaire (par exemple : Tout savoir, Toute l'année de CM1, Mon année de $C M 1$, etc.). Les 26 produits étudiés sont destinés à des élèves scolarisés du CE2 à la $5^{\mathrm{e}}$, soit les mêmes niveaux de classe que l'enquête précédente sur les manuels, pour comparer les activités supposées pour lire et étudier, dans la classe et dans la famille (pour l'école). Dans ce cas, l'usage des supports n'a pas été étudié dans l'espace familial.

Si la problématique s'inscrit dans une perspective sociologique, l'analyse mobilise des apports d'autres disciplines: études littéraires, narratologie, sémiologie, ingénierie didactique (avec des "analyses a priori» de la lecture sollicitée et possible sous conditions), didactique de la lecture, anthropologie de l'écrit et histoire «du livre au lire» (Chartier, 2003 [1985]). En effet, ces domaines de connaissance ont objectivé des changements dans la manière de produire de l'écrit et de lire, qui permettent de distinguer plusieurs critères dont trois (successivement traités dans les parties suivantes) ont été considérés comme pertinents pour étudier les manuels et les albums, tout en les "sociologisant». En effet, si ces résultats désignent des évolutions, les études littéraires par exemple focalisent sur la dimension " créatrice » et "innovante » de ces dernières et de ce qui est sollicité du lecteur supposé. Mais le lecteur effectif enfantin ne peut pleinement profiter des modes de lecture les plus élaborés qu'à certaines conditions de maîtrise du code culturel, ou de cadrage, soit par des signes explicites de l'écrit, soit par un adulte qui guide la découverte des nouvelles manières de lire attendues. Ainsi, nous avons procédé à une mobilisation de ces résultats d'autres disciplines en tant qu'observables dans les corpus, tout en les reconfigurant sociologiquement au prisme du soupçon d'élitisme dans la manière de les adresser à un lectorat minoritaire qui serait dans une connivence suffisante pour les identifier et s'en saisir $^{2}$. Pour chacun des trois critères qui structurent les parties suivantes, nos résultats ayant déjà été publiés séparément pour les albums et pour les manuels, nous focaliserons sur les points de comparaison entre les deux types de publications, en nous permettant de renvoyer aux publications précédentes.

\section{La dimension composite des supports : quelle articulation entre systèmes sémiotiques ?}

$14 \mathrm{Au}$ sujet des albums de littérature de jeunesse, un nombre très important de publications signalent l'importance croissante du rapport texte/image, cette dernière n'étant plus une simple illustration redondante du texte. Ces analyses, parues dès la fin des années 1970 aussi bien dans les recherches francophones qu'anglophones concluent que cela implique que l'enfant, avec l'aide de l'adulte qui conduit le dispositif de lecture partagée (shared reading), doit prélever des indices discrets dans les images afin de formuler des liens logiques qui ne sont pas délivrées par le texte du livre afin d'expliquer l'histoire (Chamboredon \& Fabiani, 1977a, 1977b ; Holdaway, 1979; Heath, 1982, 1983). Si les recherches à orientation sociologique posent de suite la question des dispositions nécessaires pour socialiser à une telle manière de lire, elles vont être peu nombreuses, tandis que dans la durée vont se développer des études (littéraires, sémiologiques) qui vont décortiquer les nouvelles productions d'albums de plus en plus sophistiquées (Alary \& Chabrol-Gagne, 2011; Van der Linden, 2006). Mais ces travaux centrés sur l'aspect littéraire et narratif et sur la créativité, donnent souvent une vision 
positive des évolutions dans les albums, en rupture avec la production des décennies précédentes, dont les significations étaient délivrées explicitement par l'ouvrage et qui ne faisait pas appel à l'analyse du lecteur. Ce faisant, l'évolution à ce sujet est souvent postulée plutôt que démontrée par comparaison. De plus, elle se focalise sur les nouveautés adressées au lecteur supposé en se posant rarement la question des lecteurs effectifs, ce qui va davantage être pointé dans les travaux sociologiques et didactiques depuis le début des années 2000. Nous avons donc repris les critères d'analyse entre texte et image pour étudier l'évolution des manières de lire sollicitées par les albums et ce qu'elles supposent comme dispositions chez les lecteurs ; nous bénéficions alors des critères précis tout en les sociologisant dans une approche moins enchantée.

C'est plus tardivement, à la fin des années 1990, qu'apparaissent des travaux similaires sur les relations entre systèmes sémiotiques (types de textes, photographies, schémas, cartes, graphiques, etc.) dans les manuels et dans les supports pédagogiques tels que les fiches, qui pointent le nombre important de ces systèmes au sein d'une même double page, pour faire apprendre un savoir, et qui insistent sur le fait que ces éléments ne sont plus de simples illustrations redondantes du texte de savoir, et sollicitent d'autres manières de lire (Borne, 1998; Boyzon-Fradet, 1997; Verdelhan-Bourgade, 2002 ; Vignier, 1997). À la suite des travaux systématisant ce constat de plurisémioticité (Bautier, Crinon, Delarue-Breton \& Marin, 2012) et sa présence dès la maternelle dans les fiches dont l'évolution a été retracée (Joigneaux, 2009), il semblait pertinent de comprendre les changements dans la quantité et l'organisation des éléments en présence dans une page ou double-page de manuel afin de voir les similitudes des manières de lire attendues selon les périodes avec les albums.

De 1945 à 1974, les albums présentent une relation texte-image qui est pour l'essentiel redondante (Bonnéry, 2015a). Le plus souvent, dans les ouvrages co-créés par un auteur et un illustrateur, le texte commande le récit et le dessin l'illustre au sens propre. Il arrive que le texte invite à regarder l'image, mais c'est pour y trouver confirmation de ce qui est dit ou une occasion de le visualiser. Par exemple, dans La chèvre et les biquets du Père castor (1958), le récit s'ouvre par « Il y avait une fois une chèvre blanche et trois biquets tout blancs qui habitaient dans cette petite maison-là », le déictique final invitant à regarder dans l'image et guidant l'objet du regard. Dans ce même album, l'auteur (qui signe P. François) qui es en réalité l'éditeur pédagogue P. Faucher, anticipe une difficulté potentielle de lecture chez des enfants encore jeunes: une scène en aveugle va se jouer des deux côtés de la porte de la maison fermée en clé, quand le loup va chercher à se faire passer pour la chèvre (imitation de la voix, etc.) et ainsi piéger les chevreaux. Pour guider la compréhension, les deux faces de la porte sont peintes de couleur différente, mais le repérage de cet indice n'est pas nécessaire pour accéder à la signification principale, il n'est là que pour étayer la compréhension vers une signification unique. Ainsi, l'image est presque toujours redondante du texte.

De premiers changements se manifestent durant la période, mais timidement, dans des segments légitimes de l'édition. Ainsi, dans la littérature de jeunesse francophone, Babar inaugure un format de narration (Nières-Chevrel, 2009) : l'utilisation de l'espace de la double page en grand format est particulièrement novatrice, commençant à exploiter pour la narration les possibilités des rapports entre le texte et l'image. Et si pour l'essentiel l'image « illustre » encore le texte, celui-ci peut inviter ponctuellement à adopter un regard sur les images qui ne se limite pas au prélèvement d'une représentation de la scène décrite verbalement. Une double page dans Le château de 
Babar, en 1961 montre ainsi les personnages de la famille du personnage éponyme dans des occupations diverses au sein du parc du château, le narrateur décrit de manière redondante les actions de chaque personnage, sauf pour le signe Zéphyr, évoqué en dernier : « et Zéphyr... fait des bêtises ! ». Lesdites bêtises ne sont pas explicitées par le texte, mais on peut considérer que celui-ci indique explicitement qu'il faut prélever un indice dans l'image où l'on voit au premier plan (et à droite de l'image, correspondant au sens de lecture puisque cet aspect de la description intervient à la fin du texte) le personnage concerné qui grave au couteau son prénom dans l'écorce d'un arbre.

C'est aussi dans cette période que paraît, d'abord en Italie puis en France, Petit Bleu et Petit Jaune, de L. Lionni, où des tâches de couleurs primaires anthropomorphisées et éponymes deviennent vertes lorsqu'elles se serrent. Ceci n'est que suggéré par le texte, intelligible seulement par le recours à l'image et à la mobilisation d'une connaissance technique sur les mélanges de couleurs: "Mais voilà que dans l'embrassade ils deviennent verts! Alors ils comprennent ce qui est arrivé $»^{3}$.

Contrairement à l'exemple précédent de Babar, l'activité cognitive de mise en correspondance du texte et des images, de prélèvement d'indices pour produire une signification, est ici obligatoire pour accéder au sens principal de l'histoire. L'image n'est plus à contempler seulement pour sa beauté ou pour mieux visualiser ce que le texte explique, elle est à questionner, à considérer comme ressources d'indices pour une signification autre. Et de fait, le lecteur n'est plus celui qui reçoit cette dernière, mais celui qui la cherche, qui la produit, qui se doute qu'elle a été distillée par l'auteur à l'attention de ceux qui sauraient la débusquer. Mais ces albums que nous désignerons par « implicites » apparaissent durant cette période en nombre très limité, et sont tous publiés par des éditeurs en quête de légitimité artistique. L'immense majorité de la production reste très « explicite » : le texte dit l'essentiel de ce qui est à comprendre.

Un constat assez proche peut être dressé pour ce qui est des manuels, durant la même période, à savoir celle précédant la création du collège unique où l'école maternelle française a été très innovante, intégrant les préoccupations culturelles des nouvelles catégories moyennes intellectualisées (Chamboredon \& Prévot, 1973). Si l'on note progressivement une utilisation de l'espace graphique en double-page plutôt qu'en page simple, en empruntant ce qui s'est développé préalablement dans les albums narratifs, le nombre d'images ou de documents en plus du texte reste restreint (deux en moyenne par double page). Et les systèmes sémiotiques sont peu variés, du fait de la rareté des graphiques, des tableaux de données : on relève surtout des photographies et des schémas pour donner à voir l'objet de savoir, voire des cartes. Surtout, ce ne sont pas des documents à étudier pour en tirer des informations: pour ce qui est des photographies, leur présence est surtout illustrative; et les cartes en histoire ou schémas en sciences synthétisent des informations déjà données dans le texte tout en les organisant dans l'espace ou dans le temps (pour montrer des déplacements ou des évolutions). Dans les manuels les plus proches de la "pédagogie active", il arrive que les consignes demandent d'observer un schéma ou une carte, mais alors la réponse est toujours donnée par le texte, donnant à voir ce qui est à prélever comme information et le type de regard d'étude du document attendu. Et nous n'avons pas trouvé d'activité demandant de comparer des documents, par exemple entre châteaux du Moyen Âge et de la Renaissance : ceux-ci sont montrés séparément, sans relation, sans explication des enjeux architecturaux, associés seulement à la narration de grands faits de personnages célèbres (les victoires de Bayard, les goûts de François I ${ }^{\mathrm{er}}$ ). Les activités consistent à 
faire identifier les savoirs de façon redondante entre éléments textuels et iconographiques et à les faire répéter en vue de les faire mémoriser (Bonnéry, 2015a).

De 1975 à 1994, la part des albums qui jouent sur la relation texte-image augmente progressivement, tout en restant très minoritaire, mais vers la fin de la période, commencent à paraître des titres dont les ressorts narratifs reposent tout particulièrement sur le jeu entre textes et images, dont certains vont devenir des bestsellers des écoles primaires, et consacrer des auteurs qui, dans la période suivante, vont donner libre cours à ces jeux narratifs sur les systèmes sémiotiques: G. De Pennart, T. Ungerer, M. Ramos, etc.

Cette période est celle où les manuels voient apparaitre, avec un décalage, des phénomènes apparus plus tôt pour les albums. Ainsi, si les images illustratives restent très présentes, les documents sont de plus en plus souvent à étudier, là où cette activité restait rare dans la période précédente; et comme dans celle-ci, les significations découlant de cette étude de document sont pour l'essentiel délivrées par le texte, l'énonciation des savoirs qui découle de cette étude étant assurée par le manuel au moins comme correction de l'activité. Et ces activités portent sur des savoirs descriptifs, par exemple du lexique d'architecture permettant de distinguer un château médiéval d'un autre de la Renaissance (1986, Histoire, CE2. Paris : Magnard, p. 56-57) : en poursuivant cet exemple, le manuel demande de comparer les deux styles à partir de deux images, mais le texte contigu apporte la réponse. Ainsi, l'élève ne sachant pas bien analyser le document pour en tirer du savoir n'est pas sans solution pour mémoriser le savoir.

Dans le corpus qui va de 1995 à 2014, le jeu entre les indices sémiotiques se complique, surtout dans les publications les moins « grand public ». Par conséquent, la relation qui s'établit dans le livre entre le «narrateur textuel » et le « narrateur iconique » devient plus complexe (Nières-Chevrel 2009; Boulaire, 2011), voire contradictoire (Stephen, 1992 ; Nikolajeva \& Scott, 2001).

Par exemple, de plus en plus fréquemment, la signification de ce qui se passe à un moment du récit n'est pas explicitée jusqu'au bout dans le texte, laissant au lecteur, ou au dispositif pédagogique qui organise les formes de la lecture partagée entre enfant et adulte, le soin de compléter les signes textuels par les indices iconiques (Zevenbergen, Whitehurst, Zevenbergen, 2003 ; Whitehurst et al., 1988, 1994). Ou encore, quand une version de l'histoire est donnée par le texte, que les images viennent contredire, nuancer, ou inciter à entendre autrement que ce que la lecture "à plat " peut laisser croire (Nières-Chevrel 2009; Chartier, 2009) : par exemple Balthazar ou Je suis revenu, de G. de Pennart. D'où des textes plus allusifs, supposant la mise en relation avec des indices non textuels, et des activités cognitives qui ne soient plus exclusivement celles de compréhension du sens délivré par l'oralisation du texte.

Ces évolutions engagent ainsi un autre type d'échanges lors de la lecture partagée (Golden, 1990 ; Grossmann, 1996 ; Sipe, 1998 ; Boiron \& Bensalah, 2004 ; Boiron, 2006 ; Leclaire-Halté, 2008; Boiron \& Rebière, 2009 ; Jaubert \& Rebière, 2010) et, in fine, un autre type de lecture où l'on ne forme plus à se laisser porter par l'histoire, mais à la questionner. Ces constats convergent avec les résultats antérieurs des recherches anglo-saxonnes sur les nouvelles formes de littératie dans les albums (Williams, 1995, 1999, 2000 ; Joigneaux, 2013).

Mais on assiste à une segmentation encore plus nette du marché entre albums, puisque ces constats valent pour les albums des éditeurs les plus reconnus dans le domaine 
culturel (ils ont remporté de nombreux prix et sont mis en avant par les libraires et bibliothécaires) et dans le domaine scolaire (les listes d'ouvrages conseillés en 2002, 2007 et 2013 leur font la part belle). Tandis que les productions destinées au grand public continuent à être racontées avec une relation texte-image très redondante : elles sont diffusées dans les réseaux de distribution conjoints avec les produits alimentaires ou au travers de séries dérivées de produits audiovisuels, et parmi lesquels on trouve les plus fortes ventes.

Les manuels scolaires montrent des évolutions en partie similaires. En effet, les systèmes sémiotiques sont présents dans chaque double-page en nombre supérieur à la période précédente, et les éléments textuels et iconiques sont souvent à interroger. C'est par exemple le cas quand un personnage historique est cité, son point de vue n'étant pas à prendre pour argent comptant, mais à resituer selon la place qu'il occupe dans un conflit, grâce à la confrontation aux indices présents dans les autres systèmes sémiotiques présentés à côté (Bautier, Crinon, Delarue-Breton \& Marin, 2012 ; Bonnéry, 2015a). Qu'il s'agisse donc d'albums ou de manuels, l'élève doit articuler ces différents systèmes sémiotiques pour produire des contenus, construire des savoirs, qui ne sont donc pas souvent délivrés explicitement. Ceci implique une "culture du document " à interroger (Olson, 1998 [1994]; Bautier \& Rayou, 2009), que manifestent surtout les élèves des établissements au recrutement plus favorisés (Delarue-Breton \& Bautier, 2015). Au demeurant, un certain nombre de consignes dans les manuels ont l'air de focaliser l'attention de l'élève pour analyser ces images vers des constats factuels, simples. Mais l'analyse montre que ces consignes sont désormais les premières d'une salve de questions, dont l'enchaînement tacite conduit en réalité à ce que les premiers constats alimentent les réponses aux questions suivantes, les élèves gagnant à anticiper cet enchaînement pour que les constats permettent cette remontée en généralité vers les savoirs. Cette structuration du manuel conduit souvent dans nos observations (mais pas toujours) à pousser les enseignants à une division sociale du travail intellectuel dans la classe, les élèves les moins initiés étant d'abord sollicités pour formuler les constats, que les plus connivents pourront ressaisir dans les réponses aux questions suivantes (Bonnéry, 2011).

À cette segmentation des tâches qui sont destinées aux différents types sociaux d'élèves, s'ajoute un dédoublement segmenté du marché des manuels : d'une part ceux utilisés à l'école, et d'autre part, les manuels parascolaires, destinés aux familles pour étayer les devoirs, et qui doivent se mettre à portée de tous les parents. En restant sur l'exemple précédent de la compréhension des styles différents entre époques, dans Je comprends tout (2009, Je comprends tout CM1. Nathan, p. 164-165), le dernier exercice de la double page présente deux tableaux de peintures, le «A » Diane chasseresse, et le « B » une icône de "la Vierge et l'enfant Jésus » entourés d'anges. Ces tableaux sont suivis de huit phrases à trous. Or, il n'est nul besoin de recourir aux caractéristiques de l'art au Moyen Âge ou à la Renaissance pour réaliser l'exercice. " On voit une femme nue et un chien... Le fond de la peinture est doré... La femme porte un enfant: ils sont tous habillés... »

Pourtant, des savoirs sont distillés, mais ils ne sont pas nécessaires pour avoir «juste " à l'exercice. C'est le cas dans la phrase « Le document... est une peinture religieuse : elle date du Moyen Âge ». La Vierge et l'enfant, donc la dimension religieuse, sont faciles à identifier pour toutes les familles, le fait que la peinture soit du Moyen Âge n'est pas un critère nécessaire pour réaliser l'exercice. C'est par contre un savoir potentiellement 
identifiable pour ceux qui sauront décoder le double objectif scolaire tacite, d'une part d'apprendre à étudier un document, et d'autre part de comprendre caractéristiques de chacun des styles des deux périodes dans la désignation par ces phrases à trous. Car l'exercice est appréhendable à deux niveaux. Le premier, le plus simple, permet de se mettre en règle avec l'exigence de travailler à la maison, en mobilisant des activités cognitives parmi les plus accessibles: retrouver dans les images des informations explicites du texte; l'élève peut remplir les trous sans lien avec la comparaison de période historique ou de mouvement artistique, donc sans « étudier » le document audelà des informations données par les consignes (reconnaitre des personnages nus ou habillés, un thème religieux ou pas). Pourtant, la double page se conclut par «Bravo! Maintenant, tu sais découvrir un mouvement artistique ». Le deuxième niveau invite le parent à se saisir de l'exercice en formulant des caractéristiques spécifiques de chacun des deux styles, comme les « corrigés/guide des parents » le stipulent. L'imitation de la démarche «constructiviste» et de l'activité d'étude de documents n'est ici qu'apparente.

\section{Des supports sollicitant la mise en relation d'éléments disjoints pour les organiser} implicite en cela que la signification même du livre n'est pas délivrée: il faut la produire, par exemple en mettant en discours ce qui se dégage d'un enchainement d'événements, en réorganisant les étapes de l'histoire, ou en revisitant ce que l'on croyait avoir compris (voire le titre de l'album, qui peut s'avérer polysémique) à la fin de la lecture grâce à un indice nouveau. Les éléments narratifs ne sont pas forcément donnés à voir dans une organisation qui fournit explicitement un sens cohérent avec des interdépendances, par exemple cause / conséquence: l'enfant lecteur, avec l'étayage du dispositif de lecture, est sollicité pour "expliciter les liens» (Crinon, 2008). De plus, certaines fins sont volontairement ouvertes, invitant souvent le lecteur à conclure lui-même, à dégager sa propre morale, qui est donc moins fréquemment délivrée par le narrateur textuel ou le personnage qui fait figure d'adulte ou d'autorité (Ewers, 1998 [1996] ; Gee, 1990 [1996] ; Nières-Chevrel, 2009). Ces constats fournis par des études littéraires sont vus comme un gage de richesse artistique, d'une littérature de jeunesse affranchie du poids des obligations éducatives de moralisation et d'édification. Si leurs indicateurs ont guidé notre regard, c'est en nous demandant à quelles conditions ces lectures élaborées étaient possibles. Mais ces albums restent très 
minoritaires, la plupart de la production délivrant des trames narratives relativement explicites.

Le nombre progressivement croissant d'éléments présents dans la double-page requiert une organisation plus élaborée, mais elle reste, dans la quasi-totalité des cas, très guidée et linéaire. Seule la toute fin de la période amorce des changements décrits dans la période suivante.

De 1995 à 2014, les albums aux trames narratives dont la continuité entre les étapes n'est pas explicite voient leur nombre grandir tout en restant minoritaires. Se développent dans ce cadre des récits volontairement polysémiques, invitant le lecteur à formuler des versions contradictoires (Bonnéry, 2015a), ce qui suppose donc la présence d'adultes qui initient et guident vers ce type de lectures menant l'enquête sur des sens pluriels.

Les évolutions survenues dans l'organisation narrative des albums se retrouvent de plus en plus systématiquement dans l'organisation des double-pages de manuels, « en mosaïque » (Laborde-Milaa, 2007 : p. 35), ou « éclatée », qui rompt avec l'organisation « linéaire » des manuels qui prévalaient depuis un siècle (Vignier, 1997). Nécessité est faite à l'élève, pour apprendre, de « comprendre l'organisation d'une double page [car le support] se présente toujours sous forme d'un écrit pluricodé, éclaté, dont la lecture ne peut s'effectuer de manière linéaire » (Boyzon-Fradet, 1997 : p. 72-73). De plus, il ne s'agit pas seulement de « lire» des informations disponibles, mais de les produire dans chacune des sections de la double-page, puis de les mettre en relation pour construire des savoirs. Par exemple, dans Les ateliers Hachette - Hstoire (2009, p. 60-61), le concept de «Renaissance " doit être construit à partir de la confrontation des activités à conduire préalablement et réparties dans différentes zones (non hiérarchisées et nonchronologisées) de la double-page, censées conduire à comprendre ce qu'est ce mouvement en architecture, en littérature, en philosophie, dans les sciences et techniques, et ce, en Italie puis en France : les textes de savoirs ne sont que parcellaires car les double-pages ne donnent le plus souvent qu'un "résumé » succinct, c'est à l'élève d'organiser l'ensemble et de produire un discours de mise en cohérence, et à l'enseignant de proposer le cadre qui le permet, peu aidé par le manuel.

Pour leur part, les manuels parascolaires montrent sur ce critère également un abaissement des exigences par rapport aux supports utilisés en classe : si différentes portions de double-pages existent, elles sont assez autonomes puisque les contenus qui se dégagent de chacune ne sont pas à mettre en relation entre eux. Ils illustrent simplement un aspect du texte d'introduction.

\section{Une lecture qui articule le support avec des connaissances culturelles}

Enfin, nombre d'analyses d'albums ont souligné la richesse des créations actuelles en termes d'intertextualité et d'intericonicité (Connan-Pintado, Gaiotti \& Poulou, 2008), tout particulièrement dans les « contes détournés » (Tauveron, 2009; Connan-Pintado, 2009), lesquels nécessitent de comparer la version originelle du conte à l'histoire racontée dans l'album qui prend sciemment le contrepied de certains aspects. Cela nous a conduit à étudier plus spécifiquement les allusions culturelles opérées dans nos 
corpus, en interrogeant la définition sociale du lecteur qui est supposé connaitre ces œuvres ou pratiques ainsi que sur ce qu'est le lire attendu.

De 1945 à 1974, les albums font peu allusions à des œuvres d'art, et lorsque c'est le cas, l'inférence n'est pas nécessaire pour comprendre la trame principale de l'histoire (Bonnéry, 2010a, 2010b). Les personnages archétypiques se comportent de la manière attendue (Connan-Pintado, 2009 ; Boutevin \& Richard-Principalli, 2008) et si le lecteur a besoin d'avoir construit ce qu'est un personnage pour en comprendre le comportement et les états mentaux (Glaudes \& Reuter, 1996; Cèbe, 2010), l'album ne joue pas à détourner les codes. Même les rares exceptions dans des albums précurseurs (comme Le géant de Zéralda sur lequel nous allons revenir) délivrent une première signification explicite. En résumé, un enfant accompagné d'un adulte sans connaissances littéraires ou de la culture cultivée peut accéder aux significations principales des ouvrages.

Le constat est le même dans les manuels scolaires de cette période, les allusions aux œuvres d'art sont rares, sauf en histoire. Et dans ce cas, les tableaux et statues sont en nombre restreint, dans une optique soit illustrative (le portrait officiel de François I ${ }^{\text {er }}$ ou Napoléon Bonaparte servant à se faire une image des souverains, ne pas les regarder ou ne pas les identifier comme peinture a peu d'incidences directes au regard des enjeux didactiques) soit expositive : le manuel désignait alors la reproduction comme une œuvre de référence pour la période, voire signifiait que le souverain mettait en scène son pouvoir, mais en délivrant peu, et de façon "allusive ", les savoirs qui en permettaient la maitrise dans le domaine artistique et/ou historique (Bonnéry, 2018).

De 1975 à 1994, la narration des albums continue dans l'immense majorité des cas à s'adresser à un lecteur supposé qui n'a pas besoin de connaissances culturelles pour profiter des histoires. Mais en toute fin de cette même période, commencent à se développer des récits qui détournement les archétypes, invitant à une lecture qui, dans le décalage entre ce que les personnages sont supposés faire et ce qu'ils font, ouvre à des significations nouvelles qui sont suggérées assez implicitement. Emerge ainsi un genre fondé sur le détournement des codes archétypiques, où la connivence tacite supposée avec les allusions culturelles est de plus en plus indispensable. Ainsi, quand dans la période précédente T. Ungerer en 1967 délivrait dans Le géant de Zéralda une première histoire explicite où un ogre devient rassasié grâce aux talents de cuisinière d'une fille dont il devient amoureux et qu'il épouse après avoir rasé sa barbe, d'autres significations pouvaient être produites, mais sans obligation, en reconnaissant des allusions à des tableaux de P. B. Bruegel, ou en identifiant le détournement du caractère archétypique de l'ogre vorace et inamendable. Un nouveau pallier est franchi par exemple en 1994 avec G. de Pennart, dans Le loup est revenu où les allusions à des contes et fables est bien plus nécessaire pour comprendre la trame de l'histoire, et pourquoi les personnages agissent comme ils le font. Ces albums diffusés par les éditeurs du pôle artistique et littéraire sollicitent ainsi une manière de lire "en palimpseste » (Genette, 1982) qui est de moins en moins une possibilité parmi d'autres, et plutôt une nécessité pour s'approprier l'ouvrage. Ces albums restent très minoritaires, mais leur succès en fin de cette période va ouvrir la voie à un accroissement des jeux narratifs dans la période suivante.

Pour leur part, les manuels, entre 1975 et 1994, voient peu de changements sur ce critère, si ce n'est que la place des œuvres d'art dans les manuels d'histoire augmente légèrement en cours de période, et qu'elles commencent à apparaitre dans d'autres disciplines, mais en restant essentiellement illustratives : leur connaissance préalable 
n'est pas requise, le fait de les identifier en tant qu'œuvre d'art ne change pas la signification de la double page. C'est dans la période suivante que des changements vont survenir à ce sujet dans les manuels, intégrant les manières de lire apparues dans les albums légitimes.

En effet, entre 1995 et 2014, le jeu narratif du détournement des codes littéraires est exploré dans toutes ses dimensions par une diversité d'auteurs, et les récits fondés sur des références culturelles supposées être connues se multiplient : il faut connaitre une comptine pour comprendre que le récit est fondé sur l'histoire d'enfants qui jouent au loup ; il faut savoir que des toiles de maîtres existent pour saisir le récit du livre ; il faut connaître ce que les études littéraires nomment les « coups narratifs » (Tauveron, 2009) qui sont détournés. On s'attend par exemple à voir opérer le ressort du "trompeur trompé » ou de l'arrivée d'un personnage effrayant dans telle situation où l'image et le texte ont créé une ambiance inquiétante... pour déboucher sur un événement qui rompt avec cet horizon d'attente. Ainsi, le lecteur est invité à lire en ayant en tête les intentions des auteurs, de manière réflexive par rapport à ses propres habitudes littéraires et culturelles, au travers de postures d'enquêteur sur les significations possibles.

Si ce type d'albums se développe, c'est en lien avec l'essor du marché de la littérature de jeunesse cultivée, permettant à des auteurs et dessinateurs aux ambitions artistiques de produire pour l'enfance (Fabiani, 1995), mais cette production reste minoritaire par rapport à la masse des albums « grand public » qui perdurent et se développent grâce à l'élargissement des pratiques de lectures partagées dans toutes les classes sociales (Octobre, Détrez, Mercklé, \& Berthomier, 2010 ; Bonnéry, 2015b). Le même constat de segmentation du marché entre les albums, sur ce critère également, se confirme.

Dans la même dernière période, les manuels d'histoire regorgent d'œuvres, au moins une et souvent bien davantage dans chaque double page. Et il ne s'agit plus désormais, sauf exception, d'une fonction illustrative, et moindrement de faire seulement connaitre des œuvres célèbres d'une époque, même si cet objectif est de toute évidence sous-jacent aussi : ces peintures et sculptures doivent nécessairement être étudiées en tant que document, identifiées et resituées dans leur contexte historique, et à un degré moindre artistique, pour permettre de comprendre la leçon. Par exemple dans les doubles pages sur la Renaissance, la nouvelle place de l'être humain et la nouvelle vision du monde sont à "construire" à partir de plusieurs dimensions, parmi lesquelles figurent les beaux-arts et le développement du courant humaniste en philosophie et en littérature. Ces arts sont alors à la fois des objectifs de connaissances factuelles et des moyens pour s'approprier des concepts historiques, ce qui n'était jamais le cas il y a un demi-siècle. Cette double dimension se traduit par exemple selon les manuels par ceux qui évoquent l'œuvre de Michel-Ange au plafond de la chapelle Sixtine, ou le développement de la pratique du portrait en peinture: mais dans ce dernier cas, c'est rarement La Joconde qui figure dans la plupart des produits éditoriaux, alors que c'est le seul tableau que tous les élèves connaissent unanimement. Certes, le but n'était pas, autrefois, de préparer tout le monde à des études longues, mais il semble que cette "préparation" soit, de nos jours, soumise à bien des conditions pré-requises, car la charge cognitive semble lourde pour identifier des œuvres typiques en lien avec les savoirs de l'art (la période, le courant, l'artiste, etc.) et simultanément mobiliser ces connaissances pour un travail conceptuel sur le renouveau de l'art en lien avec celui de la pensée à une époque historique. Bien sûr, 
l'objectif des concepteurs de manuels doit probablement aussi résider dans la volonté de faire connaitre d'autres œuvres. Mais ces évocations très allusives, et visant beaucoup d'objectifs simultanés, semblent peu à même de permettre aux élèves noninitiés d'identifier des œuvres en tant qu'elles font partie d'un genre de tableaux, le portrait, ni de comprendre que le développement de ce dernier lors de la Renaissance fait partie de changements historiques où l'individu prend une place croissante dans la bourgeoisie (Bonnéry, 2018).

Si c'est particulièrement vrai pour l'histoire, le constat vaut également pour des manuels d'autres disciplines, traduisant les injonctions à l'approche interdisciplinaire. Par exemple, les manuels de mathématiques, à un degré moindre en quantités d'apparitions, mobilisent régulièrement des tableaux de peinture pour étayer des leçons, ou dans des exercices, par exemple pour ce que notre recherche a relevé le plus souvent: sur le "nombre d'or» (Botticelli, La Naissance de Vénus), sur la symétrie (Mondrian), la géométrie dans l'espace (Vasarely), etc. Le type de lecture sollicitée consiste alors tout à la fois à mobiliser des connaissances supposées être au moins partiellement présentes (la pratique d'amateur d'art, non partagée par toutes les familles), pour les déplacer et les reconfigurer au service d'un autre apprentissage, comme s'il allait de soi de s'approprier un savoir et immédiatement le dépasser avec un regard distant sur celui-ci.

47 Les manuels parascolaires montrent un net abaissement des exigences en la matière, car les connaissances culturelles y sont moins requises. Soit elles sont illustratives (le portrait de François I ${ }^{\text {er }}$ dans l'exemple précédent sur la Renaissance), soit elles sont explicitées, voire, comme on l'a vu dans le même exemple, elles sont évoquées sans être l'objet de réflexion de l'exercice, les consignes pour observer le document ne portant pas sur la distinction entre peinture médiévale et renaissance, ces informations venant « au passage».

\section{De nouvelles manières de lire : pour qui ?}

La comparaison diachronique des manières de lire sollicitées par les albums et les manuels montre d'abord que certaines évolutions surviennent d'abord dans les albums et particulièrement dans le pôle de cette production le plus distingué sur le plan littéraire et artistique, avant de se retrouver dans les manuels scolaires. Cette apparition se situe dans les périodes où surviennent de nouvelles réformes de démocratisation des études : si nous n'établissons pas de rapport de causalité, il serait assez logique que les décisions de conduire davantage de jeunes dans des scolarités plus longues aillent de pair avec la volonté de les préparer aux manières de lire les plus élaborées, qu'ils vont rencontrer dans les cycles suivants. C'est aussi la période où l'école maternelle, et l'élémentaire, intègrent des pratiques sociales qui font partie de la culture des classes dominantes ainsi que des fractions intellectualisées du salariat: elles sortent de leur ancienne mission de scolarisation minimale des classes populaires. Par conséquent, en devant préparer l'ensemble d'une génération à une scolarité plus longue, donc à analyser des documents et pas seulement à recevoir des informations, l'écrit a intégré simultanément des conceptions du lire plus artistique et/ou plus exigeant, plus réflexif. Si on peut en trouver des traces dans les albums à diffusion plus "grand public ", ces évolutions restent rares, de même qu'elles sont contenues, voire contournées dans les manuels parascolaires, où elles sont intégrées a minima puisque 
ces derniers sont supposés aider à utiliser les manuels en classe, tout en étant accessibles aux parents. Ce sont les albums narratifs des éditeurs légitimes dans les mondes éducatifs et artistiques qui montrent cette évolution de la façon la plus manifeste dès l'âge de la maternelle, de même que les manuels scolaires que ces enfants rencontrent quelques années après, en élémentaire, même si le contenu est un peu plus guidé quand même pour enseigner des savoirs que pour les fictions.

Cette lecture très élaborée attendue par ces supports n'est plus celle qui se laisse guider par le texte et les consignes explicites, mais qui repose sur la conscience que tout n'est pas dit parce que des significations sont à produire. Ainsi, se sont progressivement développées des formes d'écrit qui disent moins au lecteur le contenu du message, mais organisent le support pour conduire le lecteur à penser lui-même à partir d'éléments qui lui sont fournis. Ces supports sollicitent des activités cognitives telles que nous les avons définies précédemment, c'est-à-dire non pas de recevoir ces dernières toutes prêtes, mais de les formuler en menant l'enquête par le prélèvement d'indices : le texte ne dit pas tout, il faut le confronter à d'autres systèmes sémiotiques, et lui-même est à interroger pour ce qu'il dit, ou pas, ou laisse entendre; de plus, ce qui est «lu» et « compris » à un moment doit être confronté avec d'autres parties de la double-page de manuel et/ou de l'album; et ce qui est lu renvoie de plus en plus souvent à des œuvres culturelles dont la connaissance peut éclairer autrement la lecture.

Les constats de cette recherche font écho à ce qu'avance D. Olson sur un empan temporel beaucoup plus large. Selon lui, si l'invention de l'écriture dans l'Antiquité a eu d'abord pour fonction de soulager la mémoire, et que ce faisant l'écrit a permis le développement de nouvelles formes de pensée rationnalisée (Goody, 1978 [1977), une autre étape dans ce développement semble avoir été franchie au XVII ${ }^{\mathrm{e}}$ siècle, quand les artistes et savants, après l'invention de l'imprimerie, se sont engagés dans une vaste entreprise dans laquelle ils croyaient représenter le monde.

Mais leur tentative d'atteindre à l'objectivité a eu d'autres conséquences. La différence entre ce qui est « dans le monde » (et que l'on peut voir) et ce que l'on y «voit» est de plus en plus aiguë [...] Le monde papier n'a donc pas simplement donné le moyen d'accumuler et de stocker ce que tout le monde savait. Il a permis d'inventer des moyens conceptuels pour coordonner les bribes de savoirs [...] puisées à de nombreuses sources, et de les inscrire dans un cadre de référence commun et pertinent. (Olson, 1998 [1994], p. 258-259)

51 Et une nouvelle étape a selon lui été franchie au $\mathrm{xx}^{\mathrm{e}}$ siècle, avec la création de nouveaux types de signes et de textes :

Ces nouveaux textes se sont développés grâce à un changement d'attitude par rapport aux signes. Rappelons-nous l'épigramme de Gilson, selon lequel jusqu'à Giotto, les peintures étaient des choses, alors que, de Giotto jusqu'à Cézanne, elles étaient des représentations des choses. [...] Cette attitude nouvelle [...] n'a pas seulement changé la manière de lire (selon le sens littéral), mais aussi la manière d'écrire, pour créer des « représentations ». (Olson, 1998 [1994], p. 222).

52 Cela a des conséquences sur l'école qui, selon Olson, doit préparer les élèves à maîtriser « les pratiques littératiennes de la société dominante » (Olson, 2006, p. 87):

[l']on peut montrer aux enfants comment comprendre un texte, exactement comme on doit leur montrer comment regarder une image s'ils doivent reconnaître ce que l'écrivain ou le peintre ont fait, et avec quelle intention, pour atteindre quel effet. [...] La compréhension et la production de textes exigent que l'on traite à la fois le contenu et la valeur d'illocution, ce qui est dit et la manière dont il faut le comprendre. Le lecteur et le scripteur expérimentés sont conscients de ces deux 
dimensions. Le lecteur expérimenté peut reconnaitre l'esprit derrière l'écriture, ainsi que l'esprit du lecteur supposé que le scripteur a en tête. Ces deux esprits, le lecteur doit les coordonner avec le sien propre. (Olson, 1998 [1994], p. 284). l'un des enjeux de l'École, face à de nouvelles formes de lectures qui se sont développées, tout en laissant dans l'ombre que si l'« on peut » montrer aux enfants, cela implique des supports adaptés pour cet enseignement progressif, alors que nos résultats montrent plutôt que cette «littératie étendue" (Bautier \& Rayou, 2009; Bautier, Crinon, Delarue-Breton \& Marin, 2012) s'est imposée assez rapidement sur le mode de l'évidence, comme si les enfants de tous les milieux étaient de plain-pied avec ces manières de lire que leurs parents n'ont généralement pas connues en tant qu'élèves. d'enfants, cela ne recouvre pas non plus les mêmes réalités dans l'espace social. Si ce sont les albums du pôle cultivé de l'édition qui ont les premiers manifesté ces changements dans les manières de lire, c'est qu'il s'agit là d'un type de pratiques résultant du recrutement d'auteurs et d'artistes créateurs, ne se limitant pas à des récits éducatifs, mais à transposer en direction du lectorat enfantin des manières de raisonner et de jouer avec le lecteur ou le spectateur qu'ils ont profondément intégrées à leur démarche. La force économique de ce secteur de l'édition a pu les attirer et les fidéliser (Fabiani, 1995; Gagey, 2007 ; Montmasson, 2016). L'album, après avoir été porté dans les années 1960 par les "nouvelles classes moyennes" pour qui les «nouvelles définitions sociales de l'enfant » en tant qu'être à éduquer précocement par la socialisation culturelle et artistique et donc par la fréquentation d'objets culturels spécifiques et exigeants (Chamboredon \& Fabiani, 1977a et 1977b ; Chartier \& Hébrard, 2000), a donc ensuite été particulièrement à la pointe pour diffuser dans les mêmes classes sociales les nouvelles manières de lire. Mais ce développement a coïncidé avec la diffusion de la lecture partagée d'albums «simples » dans toutes les classes sociales, y compris les plus populaires. Si la plupart des familles lisent donc des albums à leur enfant (Octobre, Détrez, Mercklé, \& Berthomier, 2010), ce ne sont pas les mêmes supports d'activité intellectuelle, qui ne permettent ni n'encouragent les mêmes types de lectures (Bonnéry, 2015b). De plus, notre enquête sur les manières de lire dans les familles montre que le même livre est lu de façon très différentes selon les dispositions culturelles des parents, et socialise donc très différemment l'enfant lecteur, tandis que le même binôme de co-lecteurs lit assez différemment deux livres qui sollicitent des lectures différentes: les plus initiés explorent bien plus systématiquement les indices que recèle l'album, et vont même jusqu'à lire l'ouvrage "simple " en le mettant en correspondance avec d'autres, par exemple le personnage loup traité différemment (Bonnéry, 2015b). Ce qui est attendu n'est pas un lire froid et austère, où l'analyse serait totalement disjointe du ressenti, mais où la lecture "participative » et la lecture «analytique» (Lahire, 1993; Baudelot, Cartier \& Détrez, 1999; Renard, 2011) s'articulent: les éléments repérés doivent alimenter une autre appréciation, celle du lecteur qui joue avec les indices semés par l'auteur.

Cette manière de lire sous-tendue par les albums a ainsi pénétré l'école par son domaine d'interface avec la culture cultivée, ce qui semble avoir été accéléré par la légitimation de la littérature de jeunesse dans le champ scolaire, en particulier grâce aux listes officielles de livres publiées en 2002, 2007 et 2013. Si l'on ne retrouve pas exactement les mêmes titres dans les classes, les albums sollicitant les nouvelles 
manières de lire sont très représentés dans les écoles (Bonnéry, Crinon \& Marin, 2015 ; Montmasson-Michel, 2018).

Mais les usages des albums en classe semblent souvent procéder d'une division sociale des manières de lire. D'abord, les albums les plus complexes, susceptibles de faire rencontrer la manière de lire élaborée, sont moins utilisés en ZEP que dans les autres établissements, ce en quoi l'école constitue des curriculums socialement différenciés en exerçant inégalement les élèves à des niveaux de lectures différents selon leur origine sociale (Bonnéry, Crinon \& Marin, 2015). Et lorsqu'ils le sont, dans les classes hétérogènes notamment, les enseignants quand ils conduisent des séances où les commentaires encouragent les prélèvement d'indices, jouent sur l'ambiguïté de ce qu'est un groupe d'élèves: quand des questions ouvertes sont posées, les élèves les moins initiés par leur famille à la lecture savante se focalisent souvent sur des indices explicites (le texte dit que le loup a trempé sa patte dans la farine pour tromper les chevreaux, ce que l'image souligne) ou sur des constats factuels qu'ils peinent à transformer en indices signifiants. Ainsi, quand une maitresse de moyenne section demande aux élèves ce qu'ils voient sur la dernière image du Géant de Zéralda, de T. Ungerer, elle donne la parole à un enfant de famille populaire qui répète d'abord le texte (le géant a rasé sa barbe). Puis quand elle le canalise sur l'image d'un personnage enfant, l'élève repère que ce dernier tient un couteau et une fourchette dans son dos. La parole est donnée ensuite à un élève (dont l'un des parents a fait des études de Lettres) qui relie, lui, ce constat au regard que ce personnage porte sur un bébé, et à d'autres éléments de l'histoire : l'ogre assagi a un enfant qui risque de devenir un ogre à son tour. Les enseignants font souvent comme si cette énonciation d'analyse (articulant prise d'indice et production de signification) par un membre du groupe équivalait à la conduite de cette activité par tous les élèves. Il n'est presque jamais demandé au bon élève d'expliquer comment parvenir à la solution, ce qui n'aide pas les autres à transposer la lecture attendue à d'autres situations, même si dans les classes d'enseignants experts d'autres types de dispositifs ont pu être observés (Leclaire-Halté \& Maisonneuve, 2017).

57 C'est donc dans le domaine de la création littéraire et artistique que ces nouvelles modalités de lecture se sont manifestées à destination de l'enfance, avant de se traduire dans les supports d'apprentissage scolaire. Puis elle est constatée avec un décalage dans le temps dans les manuels que nous avons étudiés, destinés aux élèves du CE2 à la $5^{\mathrm{e}}$, assez simultanément avec les fiches photocopiables de l'école maternelle (Bonnéry \& Joigneaux, 2015).

Or, des recherches ont montré que les élèves, s'ils sont seuls face à ce type de manuels, sont très inégalement capables, selon leurs origines sociales, de se saisir des manières de lire attendues (Delarue \& Bautier, 2015). Et nos observations dans les classes montrent également, comme pour les albums, que lors des séances, les élèves sont mobilisés sur des types de tâches très inégales, qui les exercent donc très différemment à s'approprier les activités attendues de lecture et d'appropriation de savoir. Il faut dire que face à ces supports, la tâche de l'enseignant et celle de l'élève semblent bien plus complexes qu'autrefois si l'on ne renonce pas à la même exigence pour tous les élèves : si les textes d'après-guerre pouvaient être difficiles par leur style par exemple, ils n'étaient pas sous-tendus par des conceptions du lecteur telles que nous les avons pointées. De nos jours, nombre d'élèves du CE2 à la $5^{\mathrm{e}}$, supposés être autonomes dans le « lire ", s'avèrent en fait inégalement préparés (selon leurs socialisations familiales et 
selon ce qui a été travaillé dans leur établissement scolaire) à mobiliser les différentes manières de lire que nous venons d'évoquer, celles explicites survivant des anciennes pratiques d'une part, et celles sollicitées par les manuels de la dernière période d'autre part.

C'est en partie ce qui explique le succès des manuels parascolaires vendus aux familles, quand leurs enfants, voire les parents, sont désemparés face aux supports fournis par l'école. Si nous n'avons pas étudié leur appropriation dans l'espace domestique, on peut faire l'hypothèse que ces supports, dont les contenus sont simplifiés, et surtout dont la manière de lire attendue est plus classique, permettent d'accéder aux savoirs objectivés qui sont ici délivrés, mais n'exercent pas à la manière attendue de lire et de produire soi-même les savoirs. C'est peut-être un moyen d'expliquer l'éventail des réussites inégales au sein des catégories populaires, l'usage de ces supports parascolaires pouvant permettre des réussites partielles et donc de survivre plus longtemps dans le système scolaire sans pour autant construire les dispositions conduisant aux études les plus longues.

Si nous n'avons donc pas suivi d'enfants pendant plusieurs années et dans ses différents lieux de socialisation lectorale, tous ces résultats laissent penser que lorsqu'ils sont petits, dans leurs familles, ils sont socialisés à des manières de lire très différentes, inégalement proches de celles que sollicitent les albums les plus utilisés à l'école. Et si les séances utilisant un album enseignent peu comment lire autrement, en exploitant toutes les ressources de l'ouvrage, en laissant les élèves mobiliser les dispositions qu'ils ont construites dans la famille, les élèves s'avèrent inégalement préparés à utiliser adéquatement les supports plus scolaires, fiches en maternelle (Bautier, 2006; Joigneaux, 2009), puis plus tard les manuels scolaires en élémentaire et collège. Il semble alors que l'école, en enrôlant inégalement les élèves dans des activités qui semblent pourtant être simultanées ou conjointes, permette que chacun mobilise les dispositions familialement construites, et donc ne pas le conduire à s'approprier les nouvelles modalités de lecture. Pour rompre avec un tel curriculum différencié et différenciateur, il faudrait que la difficulté du «nouveau lire » soit objectivée et prise en compte, pour conduire à un enseignement assumé plutôt que requis.

\section{BIBLIOGRAPHIE}

ALARY, V. \& CHABROL-GAGNE, N. (dir.) (2011). L'Album. Le parti-pris des images. Clermont-Ferrand : Presses universitaires Blaise Pascal.

Baudelot C., CARTier M. \& DÉtrez C. (1999). Et pourtant, ils lisent... Paris : Éditions du Seuil.

BAUTIER, É. (dir.) (2006). Apprendre à l'école. Apprendre l'école. Des risques de construction d'inégalirés dès la maternelle. Lyon : Chronique sociales.

BAUTIER, É., BONNÉRY, S. \& CLÉMENT, P. (2017). « L'introduction en France des compétences dans la scolarité unique. Enjeux politiques, enjeux de savoir, enjeux pédagogiques et didactiques ». 
Cahiers de la recherche sur l'éducation et les savoirs 16, p. 73-93. En ligne : https://

journals.openedition.org/cres/3019.

BAUTIER, É., CRINON, J., DELARUE-BRETON, C. \& MARIN, B. (2012). « Les textes composites : des exigences de travail peu enseignées ? » Repères 45, p. 63-79. En ligne : https://journals.openedition.org/ reperes/136.

BAUTIER, É. \& RAYOU, P. (2009). Les inégalités d'apprentissage. Programmes, pratiques et malentendus scolaires. Paris : Presses universitaires de France.

BOIRON, V. \& BENSALAH, A. (2006). « Construire une méthodologie interprétative des albums à l'école maternelle : analyse des modalités de compréhension dialoguée et d'élaboration conjointe d'interprétations ». Mélanges Crapel 29, p. 11-26. En ligne : http://web.atilf.fr/spip.php? article3708.

BOIRON, V. (2006). « Le développement de l'enfant apprenti-interprète : interactions adulte-texteenfants à l'école maternelle ». Les Dossiers des Sciences de l'Education 15, p. 11-25. En ligne : https:// www.persee.fr/doc/dsedu_1296-2104_2006_num_15_1_1074.

BONNÉRY, S. (2007). Comprendre l'échec scolaire. Paris : La Dispute.

BONNÉRY, S. (2010a). « Les mises en scène dans les albums enfantins des apprentissages dans la relation entre adultes et enfants : des évolutions significatives des modalités éducatives ». Raison publique 13, p. 323-336.

BONNÉRY, S. (2010b). « “- Loup y es-tu ?. Pas exactement, c'est pour mieux te faire réfléchir, mon enfant..." Actes du congrès de l'Actualité de la recherche en éducation et en formation (AREF), septembre 2010 : Université de Genève. En ligne : https://plone2.unige.ch/aref2010/ communications-orales/premiers-auteurs-en-b/Loup\%20y\%20es-tu.pdf/view.Consulté le 3 mars 2018,

BONNÉRY, S. (2011). «Les définitions sociales de l'apprenant : approche sociologique, interrogations didactiques ». Recherches en didactiques 12, p. 65-84. En. ligne : https:// www.cairn.info/revue-recherches-en-didactiques-2011-2-page-65.htm.

BONNÉRY, S. (dir.) (2015a). Supports pédagogiques et inégalités scolaires. Paris : La Dispute.

BONNÉRY, S. (2015b). « Des livres pour enfants. De la table de chevet au coin lecture ». In P. Rayou, (dir.). Aux frontières de l'école. Institutions, acteurs et objets. Saint-Denis : Presses universitaires de Vincennes, p. 193-214.

BONNÉRY, S. (2018a). «Connivence, pré-requis et implicites : enjeux théoriques, enjeux pédagogiques ». Recherches. Revue de didactique et de pédagogie du français 70, p. 43-66.

BONNÉRY, S. (2018b). « Étudier la construction des dispositions socio-cognitives : gains transfrontaliers et frais de douanes disciplinaires ». Biens symboliques, 3. En ligne $:$ https:// www.biens-symboliques.net/293.

BONNÉRY S., CRINON J., \& MARIN B. (2015). « Les albums utilisés avec les élèves : approche quantitative des choix différenciés des enseignants de cycle 3. " Recherches en Éducation 22, p. 58-69. En ligne : http://www.recherches-en-education.net/IMG/pdf/REE-no22-2.pdf.

BONNÉRY S. \& JOIGNEAUX, C. (2015). « Des littératies familiales inégalement rentables scolairement. Ce que donnent à voir les lectures partagées d'albums de jeunesse ». Le Français Aujourd'hui 190, p. 23-33. 
BORNE, D. (1998). Programme de travail 1997-1998 Thème 2. Le manuel scolaire. Paris : La Documentation française. En ligne : http://www.soseducation.com/fichiers/ DominiqueBorne1998.pdf.

BOULAIRE, C. (2011). « Les deux narrateurs à l'œuvre dans l'album : tentatives théoriques ». In : Alary, V. \& Chabrol-Gagne, N. (éds). L'Album. Le parti-pris des images. Clermont-Ferrand: Presses universitaires Blaise Pascal.

BOULAIRE, C. (2016). Les « Petits Livres d'or». Des albums pour enfants dans la France de la guerre froide. Tours : Presses universitaires François-Rabelais.

BOUTEVIN, C. \& RICHARD-PRINCIPALLI, P. (2008). Dictionnaire de la littérature de jeunesse. Paris : Vuibert. BOYZON-FRADET, D. (1997). « Enseigner/apprendre la langue scolaire. Un enjeu fondamental pour les enfants issus de l'immigration ». Migrants-formation 108, p. 67-85.

BOIRON, V. \& REBIÈRE, M. (2009). « Quels albums pour la petite section ? Propositions de critères de choix ", Diptyque 17, p. 11-26.

CÈBE, S. (2010). « La compréhension de textes n'est pas un jeu d'enfants ». $82^{\mathrm{e}}$ colloque national Ageem. À l'école maternelle, l'école de tous les langages. Hyères-les-Palmiers, 1-3 juill. 2009. En ligne : http://ageem.fr/wcms/ftp//a/ageem.fr/uploads/cebe-2010.pdf.

CHAMBOREDON, J.-C. \& FABIANI, J.-L. (1977a). « Les albums pour enfants. Le champ de l'édition et les définitions sociales de l'enfance - 1 ». Actes de la recherche en sciences sociales 13, p. 60-78.

CHAMBOREDON, J.-C. \& FABIANI, J.-L. (1977b). « Les albums pour enfants. Le champ de l'édition et les définitions sociales de l'enfance $-2 »$. Actes de la recherche en sciences sociales 14, p. 55-79.

CHAMBOREDON, J.-C. \& PRÉVOT, J. (1973). « Le “métier d'enfant”. Définition sociale de la prime enfance et fonctions différentielles de l'école maternelle ", Revue française de sociologie, 14 (3), p. 295-335. En ligne : https://www.persee.fr/doc/rfsoc_0035-2969_1973_num_14_3_2215.

CHARTIER, A.-M. (2009). « Lire avec les enfants ». Compte Rendu de séminaire. Observatoire de l'enfance en France. 28 Janvier 2009. En ligne : http://maternelles21.ac-dijon.fr/sites/ maternelles21.ac-dijon.fr/IMG/pdf/LIRE_avec_des_Petits_obs_Enfance__No14-2.pdf.

CHARTIER, A.-M. \& HÉBRARD, J. (2000). Discours sur la lecture (1880. 2000). Paris : Bibliothèque publique d'information/Centre Pompidou.

CHARTIER, R. (2003) [1985]. « Du livre au lire ». In : Chartier R. (dir.), Pratiques de la lecture. Paris : Payot.

COLLOVALD, A. \& NEVEU, É. (2004). Lire le noir. Enquête sur les lecteurs de récits policiers. Paris : Bibliothèque publique d'information/Centre Pompidou.

CONNAN-PINTADO, C. (2009). Lire des contes détournés à l'école. À partir des Contes de Perrault. Paris : Hatier.

CONNAN-PINTADO, C., GAIOTTI, F. \& POULOU, B. (éds) (2008). L'album contemporain pour la jeunesse : nouvelles formes, nouveaux lecteurs?. Pessac : Presses universitaires de Bordeaux.

CRINON, J. (2008). « Une première culture littéraire ». Cahiers pédagogiques 462, p. 31-33.

DELARUE-BRETON, C. \& BAUTIER, É. (2015). « Nouvelle littératie scolaire et inégalités des élèves : une production de significations différenciée ». Le français aujourd'hui 190, p. 51-60. En ligne : https:// www.cairn.info/revue-le-francais-aujourd-hui-2015-3-page-51.htm.

DURKHEIM, É. (2007) [1893]. De la division du travail social. Paris : Presses universitaires de France. 
DURKHEIM, É. (1969) [1938]. L'évolution pédagogique en France. Paris : Presses universitaires de France.

EWERS, H. H. (1998) [1996]. « La littérature moderne pour enfants : son évolution historique à travers l'exemple allemand du XVIII ${ }^{\mathrm{e}}$ au XX ${ }^{\mathrm{e}}$ siècle ». In : Becchi, E. \& Julia, D. (dirs). Histoire de l'enfance en Occident. Du XVIII' siècle à nos jours. Tome 2. Trad. de l'italien. Paris : Éditions du Seuil.

FABIANI, J.-L. (1995). « Le plaisir et le devoir : remarques sur la production et la réception de livres destinés à la petite enfance ». La revue des livres pour enfants 163-164, p. 66-72. En ligne : http:// cnlj.bnf.fr/sites/default/files/revues_document_joint/PUBLICATION_3707.pdf.

FRIER, C. (dir.) (2006). Passeurs de lecture. Lire ensemble à la maison et à l'école. Paris : Retz.

GAGEY, C. (2007). «Le marché du livre jeunesse en 2006 : tendances, enjeux et problématiques ». In : La littérature jeunesse, une littérature de son temps? Actes du colloque du Salon du livre et de la presse jeunesse en Seine-Saint-Denis. Montreuil : Centre de promotion du livre de jeunesse.

GEE, J. P., (1996) [1990]. Social linguistics and literacies. Ideologies in Discourses. Londres : Falmer.

GENETTE, G. (1982). Palimpsestes. La littérature au second degré. Paris : Éditions du Seuil.

GLAUDES, P. \& ReUTER, Y. (1996). Personnage et didactique du récit. Metz : Université de Metz.

GOLDEN, J. M. (1990). The narrative symbol in childhood literature. Exploration un the construction of text. Berlin : Mouton de Gruyter.

GOODY, J. (1978) [1977]. La Raison graphique. La domestication de la pensée sauvage. Trad. de l'anglais par J. Bazin \& A. Bensa. Paris : Éditions de Minuit.

Goody, J. (1986). La logique de l'écriture. Trad. de l'anglais. Paris : Armand Colin.

GOODY, J. (2006). « La technologie de l'intellect ». Trad. de l'anglais par J.-C. Lejosne. Pratiques 131-132, p. 7-30.

Goody, J. (2007) [2000]. Pouvoirs et savoirs de l'écrit. Trad. de l'anglais par C. Maniez. Paris : La Dispute.

GROSSMANN, F. (1996). Enfances de la lecture. Manières de faire, manières de lire à l'école maternelle. Berne/Paris : P. Lang.

GROSSMANN, F. (2006). « Logiques sociales et clivages culturels dans les lectures partagées ». In : FRIER, C. (dir.). Passeurs de lecture. Lire ensemble à la maison et à l'école. Paris : Retz, p. 19-43.

HEATH, S. B. (1982). « What No Bedtime Story Means: Narrative Skills at Home and School ». Language, Society 11 (1), p. 49-76.

HEATH S. B. (1983). Ways with words. Language, life, and work in communities and classrooms. Cambridge/New York : Cambridge University Press.

HOLDAWAY, D. (1979). The foundations of literacy. Portsmouth : Heinemann

JAUBERT, M. \& REBIÈRE, M. (2010). « Former à l'enseignement de la lecture : un genre langagier professionnel et ses gestes afférents - une étude de cas ». In : Bucheton D. \& Dezutter, O. (dirs). Le développement des gestes professionnels dans l'enseignement $d u$ français : un défi pour la recherche et la formation. Bruxelles : De Boeck, p. 149-165.

JoIGNEAUX, C. (2009). « La construction de l'inégalité scolaire dès l'école maternelle ». Revue française de pédagogie 169, p. 17-28. En ligne : https://www.cairn.info/revue-francaise-depedagogie-2009-4-page-17.htm. 
JOIGNEAUX, C. (2013). « La littératie précoce. Ce que les enfants font avec l'écrit avant qu'il ne leur soit enseigné ». Revue française de pédagogie 185, p. 117-161. En ligne : https:// journals.openedition.org/rfp/4345.

LABORDE-MILAA, I. (2007). « La "fiche" dans les manuels scolaires. Un concentré clair ou opaque du pouvoir auctorial ? ». Lidil 35, p. 79-97. En ligne : https://journals.openedition.org/lidil/2213.

LAHIRE, B. (1993). La raison des plus faibles. Rapport au travail, écritures domestiques et lectures en milieux populaires. Lille : Presses universitaires de Lille.

LECLAIRE-HALTÉ, A. (2008). «L'album de littérature de jeunesse : quelle description pour quel usage scolaire ? ». In : Durand, J., Habert, B. \& Laks, B. (éds.). Actes du Congrès Mondial de Linguistique Français. En ligne : https://doi.org/10.1051/cmlf08037. Consulté le 21 mai 2014.

LECLAIRE-HALTÉ, A. \& MAISONNEUVE, L. (2017). « Les albums documentaires sur les peintres : un exemple d'interdisciplinarité au cycle 3 de l'école primaire ». Pratiques 175-176. En ligne : https:// journals.openedition.org/pratiques/3611.

MAUGER, G. (1999). «Écrits, lecteurs, lectures ». Genèses. Sciences sociales et histoire, 34, p. 144-161. En ligne : https://www.persee.fr/doc/genes_1155-3219_1999_num_34_1_1560.

MONTMASSON, D. (2016). La réception de la littérature de jeunesse par les enfants : une fenêtre ouverte sur le processus de socialisation. Thèse de sciences de l'éducation : Université Paris Descartes. En ligne : https://tel.archives-ouvertes.fr/tel-01997596/document.

MONTMASSON-MICHEL, F. (2018). Enfances du langage et langages de l'enfance. Socialisation plurielle et différenciation sociale de la petite enfance scolarisée. Thèse de sociologie : Université de Poitiers. En ligne : https://tel.archives-ouvertes.fr/tel-02462031/document.

NIÈRES-CHEVREL I. (2009). Introduction à la littérature de jeunesse. Paris : Didier Jeunesse.

OCTOBRE, S., DÉTREZ Ch., MERCKLÉ, P. \& BERTHOMIER, N. (2010). L'enfance des loisirs. Trajectoires communes et parcours individuels à la fin de l'enfance et à la grande adolescence. Paris : Ministère de la Culture. En ligne : https://www.cairn.info/l-enfance-des-loisirs--9782110975454.htm.

oLSON, D. (1998) [1994]. L'univers de l'écrit. Comment la culture écrite donne forme à la pensée. Trad. de l'anglais par Y. Bonin. Paris : Retz.

OLSON, D. (2006). « Littératie, scolarisation et cognition. Quelques implications de l'anthropologie de Jack Goody ». Trad. de l'anglais par J.-C. Lejosne. Pratiques 131-132, p. 83-94. En ligne : https:// www.persee.fr/doc/prati_0338-2389_2006_num_131_1_2119.

PRIVAT, J.-M. \& KARA, M. (éds) (2006). « La littératie. Autour de Jack Goody », Pratiques 131-132. En ligne : https://www.persee.fr/issue/prati_0338-2389_2006_num_131_1.

RENARD, F. (2011). Les lycéens et la lecture. Entre habitudes et sollicitations. Rennes : Presses universitaires de Rennes.

SEGRÉ, M. (2001). « Itinéraires de lecteurs. Note critique sur des études récentes en sociologie de la lecture ». Revue Française de Sociologie 42 (1), p. 149-164. En ligne : https://www.persee.fr/doc/ rfsoc_0035-2969_2001_num_42_1_5337.

SIPE L.R. (1998). « How Picture Books Work : A Semiotically Framed Theory of Text-Picture Relationships ». Children's Literature in Education 29, p. 97-108.

TAUVERON C. (dir.) (2009). Lire la littérature à l'école. Pourquoi et comment conduire cet apprentissage spécifique? Paris : Hatier.

VAN DER LINDEN, S. (2006). Lire l'album. Paris : L'Atelier du Poisson Soluble. 
VERDELHAN-BOURGADE, M. (2002). «Le manuel comme discours de scolarisation ». ÉLA. Études de linguistique appliquée 125, p. 37-52. En ligne : https://www.cairn.info/journal-ela-2002-1page-37.htm.

VIGNER, G. (1997). « La représentation du savoir : mise en page et mise en texte dans les manuels scolaires ». Les cahiers du français contemporain 4, p. 47-81.

WHITEHURST, G. J. et al. (1988). « Accelerating language development through picture book reading ». Development Psychology 24 (4), p. 552-559.

WHITEHURST, G. J. et al. (1994). « A picture book reading intervention in day care and home for children from low-income families ». Developmental Psychology 30 (5), p. 679-689.

WILLIAMS, G. (1995). Joint Book-Reading and Literacy pedagogy : A Socio-Semantic Interpretation. Thèse en sociolinguisitque : Macquarie University.

WiLLIAMS, G. (1999). «Children Becoming Readers. Reading and Literacy ». In : Hunt, P. (éd.).

Understanding Children's Literature. Londres/New York : Routledge, p. 151-162.

WILLIAMS, G. (2000). « Children's literature, children and uses of language description ». In :

Unsworth, L. (éd.). Researching language in Schools and Communities. Fonctional Linguistic Perspectives. Londres/Washington : Cassell, p. 111-129.

ZEVEnBERgEn, A. A., WhitehuRst, G.J., \& ZEVENBERGEN, J. A. (2003). « Effects of a Shared-Reading Intervention on the Inclusion of Evaluative Devices in Narratives of Children from Low-Income Families ». Journal of Applied Developmental Psychology 24 (1), p. 1-15.

\section{NOTES}

1. La réforme Berthoin, sous la présidence gaulliste, unifie l'école primaire et les petites classes de l'ancien lycée élitiste. Articulée aux réformes Fouchet-Capelle dans les années suivantes, elle contribue à généraliser l'accès au secondaire, tout en canalisant cette massification dans des filières séparées. La réforme Haby, sous la présidence de Giscard, dite du « collège unique » crée une seule filière en $6^{e}$ et $5^{\mathrm{e}}$, ce qui encouragera de fait l'accès massifié aux $4^{e}$ et $3^{e}$ générales, puis au lycée. Ainsi, dix ans plus tard, Chevènement, sous la présidence de F. Mitterrand, crée le lycée professionnel pour canaliser l'accès des élèves de milieux populaires dans ce second cycle de l'enseignement secondaire.

2. Sur les modalités de ces emprunts et de leurs reconfigurations pour les intégrer au raisonnement sociologique, voir Bonnéry $(2011,2018 b)$.

3. L'analyse de cet album est réalisée dans un article précurseur dont notre approche s'est beaucoup inspirée (Chamboredon \& Fabiani, 1977a et 1977b). 


\section{RÉSUMÉS}

Lire aujourd'hui à l'école élémentaire et au collège en France ne signifie plus exactement la même activité requise que celle qui était entendue par ce terme il y a un demi-siècle. C'est ce que montre une enquête sur les manuels scolaires. L'étude de leur usage dans les classes montre que ce dernier est peu enseigné, et contribue à des inégalités sociales d'appropriation entre élèves. Pour comprendre comment certains d'entre eux parviennent malgré tout à « lire » ces nouveaux types de documents, une enquête sur les albums de littérature de jeunesse pour des enfants entrant dans la lecture donne à voir des évolutions similaires du traitement de l'écrit attendu dans la même période. Et des observations de lectures partagées de ces albums en famille permettent de comprendre comment ces pratiques domestiques forment inégalement aux attendus de ce que c'est que lire aujourd'hui. Enfin, l'étude des supports parascolaires vendus aux familles montre un étayage ambigu des apprentissages.

Today, in France's elementary and secondary schools, the term reading does not exactly refer to same activity as it did half a century ago, a survey on textbooks shows that. Research on textbook use in class indicates that teachers are rarely taught how to use them, which contributes to social inequalities between students in terms of appropriation. To understand how some of them nevertheless manage to "read" these new types of documents, a survey on children's literature albums for those who are beginning to read reveals similar developments in the treatment of the written word expected over the same period. And observations of shared readings of these albums in families allow us to understand how these domestic practices provide an unequal training in terms of the expectations implied by the activity of reading as we mean it today. Finally, the study of extracurricular materials sold to families shows an ambiguous support of learning.

\section{INDEX}

Mots-clés : littéracie, lecture, manuels scolaires, littérature de jeunesse, école, famille.

Keywords : literacy, reading, textbooks, children's books, school, family.

\section{AUTEUR}

\section{STÉPHANE BONNÉRY}

Université Paris 8 Vincennes - Saint-Denis, Circeft-Escol, F-93526Saint-Denis, France 